\title{
Amelioration of hemodialysis-induced oxidative stress and fatigue with a hemodialysis system employing electrolyzed water containing molecular hydrogen
}

Hidehisa Satta' ${ }^{1}$, Tamio Iwamoto², Yuki Kawai ${ }^{2}$, Naoaki Koguchi ${ }^{1}$, Kazuhiko Shibata ${ }^{3}$, Nobuteru Kobayashi ${ }^{4}$, Mariko Yoshida ${ }^{4}$ and Masaaki Nakayama ${ }^{5,6^{*}}$

\begin{abstract}
Background: A novel hemodialysis (HD) system employing electrolyzed water containing molecular hydrogen ( $E$ $\mathrm{HD}$ ) has been developed to improve the bio-compatibility of HD. This study examined the impact of E-HD on changes in redox state during HD and HD-related fatigue.

Method: This single-arm, prospective observational study examined 63 patients on chronic HD (41 males; mean age, $72 \pm 9$ years; median duration of HD, 7 years). Redox parameters (serum myeloperoxidase [MPO], malondialdehyde-protein adduct [MDA-a], thioredoxin 1 [TRX]) during HD were compared between control HD (C$\mathrm{HD}$ ) and E-HD after 8 weeks. Fatigue was evaluated using a numerical rating scale (NRS) during the 8-week course.

Results: In C-HD, an increase in serum MPO accompanied increases in both oxidative products (MDA-a) and antioxidant molecules (TRX). In E-HD, although increases in MPO were accentuated during HD, changes in MDA-a and TRX were ameliorated as compared with C-HD. In patients who showed HD-related fatigue (47\%) during C-HD, change in MDA-a by HD was a risk factor for the presence of fatigue. During the 8 weeks of observation on E-HD, those patients displayed significant decreases in fatigue scores.

Conclusion: E-HD ameliorates oxidative stress and supports anti-oxidation during HD, suggesting improved biocompatibility of the HD system. E-HD may benefit patients with HD-related fatigue, but the mechanisms underlying changes to oxidative stress have yet to be clarified.
\end{abstract}

Keywords: Hemodialysis, Biocompatibility, Oxidative stress, Molecular hydrogen, Dialysis-fatigue

\footnotetext{
* Correspondence: nakayama@luke.ac.jp

${ }^{5}$ Kidney Center, St Luke's International Hospital, 9-1 Akashicho, Chuo-ku, Tokyo 1048560, Japan

${ }^{6}$ Research Division of Dialysis Chronic Kidney Disease, Tohoku University Hospital, Sendai, Japan

Full list of author information is available at the end of the article
}

(c) The Author(s). 2021 Open Access This article is licensed under a Creative Commons Attribution 4.0 International License, which permits use, sharing, adaptation, distribution and reproduction in any medium or format, as long as you give appropriate credit to the original author(s) and the source, provide a link to the Creative Commons licence, and indicate if changes were made. The images or other third party material in this article are included in the article's Creative Commons licence, unless indicated otherwise in a credit line to the material. If material is not included in the article's Creative Commons licence and your intended use is not permitted by statutory regulation or exceeds the permitted use, you will need to obtain permission directly from the copyright holder. To view a copy of this licence, visit http://creativecommons.org/licenses/by/4.0/ The Creative Commons Public Domain Dedication waiver (http://creativecommons.org/publicdomain/zero/1.0/) applies to the data made available in this article, unless otherwise stated in a credit line to the data. 


\section{Background}

The bio-incompatibility of hemodialysis (HD) systems, such as complement activation, enhanced expression of pro-inflammatory molecules from lymphocytes, activation of platelets, and degranulation of neutrophils, has been considered to be involved with excess comorbidities including cardio-cerebrovascular disease and HDrelated complications $[1,2]$. Current HD systems have made improvements to dialyzer materials and purification of dialysis water [3-5], but issues of bioincompatibility have not yet been fully resolved. For example, blood levels of myeloperoxidase (MPO) still increase during HD [6-8]. MPO is thought to mediate the generation of radical oxygen species in the presence of $\mathrm{H}_{2} \mathrm{O}_{2}$, which in turn exaggerates oxidative stress [9-13].

Fatigue is a crucial issue for patients receiving chronic dialysis in terms of patient-reported outcomes [14, 15]. Fatigue affects the quality of life and patient survival [16-19]. The pathological background of fatigue in dialysis patients is complex, including causes such as aging, anemia, malnutrition, inflammation, depression, and autonomic dysfunction [14, 15, 20, 21]. On the other hand, in patients receiving HD, the degree of fatigue can differ according to the presence or absence of HD, such as exaggerated fatigue on the days of $\mathrm{HD}$, and less or even no fatigue on non-HD days [20, 22]. This unique profile may indicate a potential role of HD bioincompatibility in the development of fatigue in HD patients and suggests that enhanced oxidative stress could be involved with the pathological mechanisms.

A novel HD system employing electrolyzed water containing molecular hydrogen $\left(\mathrm{H}_{2}\right)(\mathrm{E}-\mathrm{HD})$ has been developed to improve the bio-compatibility of HD [23-26]. Oxidative stress markers have already been reported to be suppressed with this system [24-26], and better clinical outcomes have been observed in patients on E-HD than in patients on standard HD [27], including the level of fatigue [28]. The aim of this study was thus to examine the impact of E-HD on changes in redox state during $\mathrm{HD}$ and HD-related fatigue.

\section{Patients and methods}

\section{Patients}

Patients comprised 63 patients on HD who were treated at Yokodai Central Clinic (Yokohama, Japan) between October 2019 and May 2020. All patients had been receiving standard HD regularly 3 times a week for $3-4 \mathrm{~h} /$ session, using a high-performance biocompatible membrane dialyzer. As of the end of November 2019, HD therapy employing electrolyzed water containing $\mathrm{H}_{2}$ (E$\mathrm{HD}$ ) as dialysis water was introduced for all patients and was provided regularly thereafter.

Patient characteristics are shown in Table 1. The 63 patients included 41 males, mean $72 \pm 9$ years, and median duration of HD was 7 years. The leading cause of underlying kidney disease was diabetic kidney disease $(43 \%)$, and $35 \%$ of patients had a history of cardiovascular disease. Informed consent was obtained from all participants, and the study protocol was fully approved by the ethics committee of Kanagawa Medical Association Ethics Review Committee (October 10, 2019). All methods were performed in accordance with the relevant guidelines and regulations.

\section{Methods}

Blood samples were obtained from all patients at 4 timepoints, before and after a session of HD within 4 weeks before the commencement of E-HD (control HD [CHD] phase) and before and after a session of E-HD 8 weeks after commencing E-HD (E-HD phase). Blood samples were centrifuged with ethylenediamine tetra-acetic acid, and all plasma was stored at $-80{ }^{\circ} \mathrm{C}$ until needed for measurements.

Preserved plasma samples were used to measure the following parameters: MPO (Human Myeloperoxidase Quantikine ELISA Kit; R\&D Systems, Minneapolis, MN, USA), thioredoxin 1 (TRX) (Human Thioredoxin Assay ELISA Kit; IBL, Fujioka, Japan), and malondialdehyde-protein adduct (MDA-adduct) (OxiSelect $^{\mathrm{mm}}$ MDA Adduct Competitive ELISA Kit; Cell Biolabs, San Diego, CA, USA).

\section{Assessments of fatigue}

Patient fatigue was evaluated using both a numerical rating scale (NRS) and our own original fatigue scale. All questionnaires were provided in written format. The NRS is a unidimensional scale with the left end anchored to "no tiredness at all (0)" and the right end to "complete exhaustion (10)." Our original fatigue scale is a four-grade self-evaluation by the patient: grade 1, no fatigue, patient acts in the ordinary way without any sense of fatigue; grade 2, mild fatigue, patient acts in the ordinary way, but feels tired; grade 3 , moderate fatigue, the patient feels tired with light work; and grade 4, intense fatigue, the patient feels very tired, and falls asleep. Measurements on our original fatigue scale were made on both a dialysis day and a dialysis-free day in the CHD phase.

Cut-off NRS scores were based on analysis of the receiver operating characteristic (ROC) curve, in which fatigue with decreased activities of daily living was defined as grade $\geq 3$ on our original fatigue scale. Logistic regression multivariate analysis was performed to estimate associations between risk of fatigue and redox parameters in the CHD phase. 
Table 1 Patients' demographics

\begin{tabular}{lc}
\hline $\mathrm{N}$ & 63 \\
Age (year old) & $72 \pm 9$ \\
Gender: male & $41(65.1 \%)$ \\
HD vintage (years) & $7(1,40)$ \\
Underlying renal disease (non-diabetic nephropathy) & $36(57.1 \%)$ \\
Comobidities & $22(34.9 \%)$ \\
$\quad$ Cardiovascular disease & $5(7.9 \%)$ \\
Malignancy & $10.5 \pm 1.1$ \\
Labolatory data & $10.0 \pm 2.1$ \\
$\quad$ Hemoglobin $(\mathrm{g} / \mathrm{dL})$ & $3.8 \pm 0.3$ \\
Creatinine $(\mathrm{mg} / \mathrm{dL})$ & $0.14(0.01,3.40)$ \\
Albumin $(\mathrm{g} / \mathrm{dL})$ & \\
C-reactive $\mathrm{protein}(\mathrm{mg} / \mathrm{dL})$ & \\
\hline
\end{tabular}

\section{Overview of the E-HD system}

Briefly, E-HD solutions were prepared as follows (Fig. 1): tap water was supply to the Electrolyzed WaterHemodialysis System (Trim Medical Institute Co., Ltd., Osaka, Japan), where water was processed using activated charcoal filtration and water softening and then electrolyzed by direct current supply to the anode and cathode electrode plates. Water on the anode side was drained, and water from the cathode side (electrolyzed water) was collected to supply the reverse osmosis module. The intensity of electrolysis was adjusted to target $\mathrm{H}_{2}$ concentration. The reverse osmosis water containing $\mathrm{H}_{2}$ (e-RO water) produced by Electrolyzed WaterHemodialysis System was supplied to prepare the HD solution. The composition of the inflow E-HD solution was the same as the CHD solution with the exception of the presence of dissolved $\mathrm{H}_{2}$ in the E-HD, and there were no differences in terms of electrolytes levels and $\mathrm{pH}$, as compared to the CHD solution, as reported elsewhere [26-28], whereas regarding $\mathrm{H}_{2}$ levels of dialysate were in the range of $41-84 \mathrm{ppb}$.

\section{Analysis}

Variables are expressed as mean \pm standard deviation (SD) or median (range), as appropriate. Statistical significance was set at the level of $\mathrm{P}<0.05$.

Comparisons between groups were performed using the paired $t$ test and non-parametric Wilcoxon paired rank test. Time courses were analyzed using repeatedmeasures analysis of variance, and Bonferroni for multiple comparison procedure. All statistical analyses were performed with SPSS version 22.0 (STATA Corporation, College Station, TX, USA).

\section{Results}

Changes in parameters are summarized in Table 2. In both CHD and E-HD phases, significant increases in MPO and MDA-a and a significant decrease in TRX

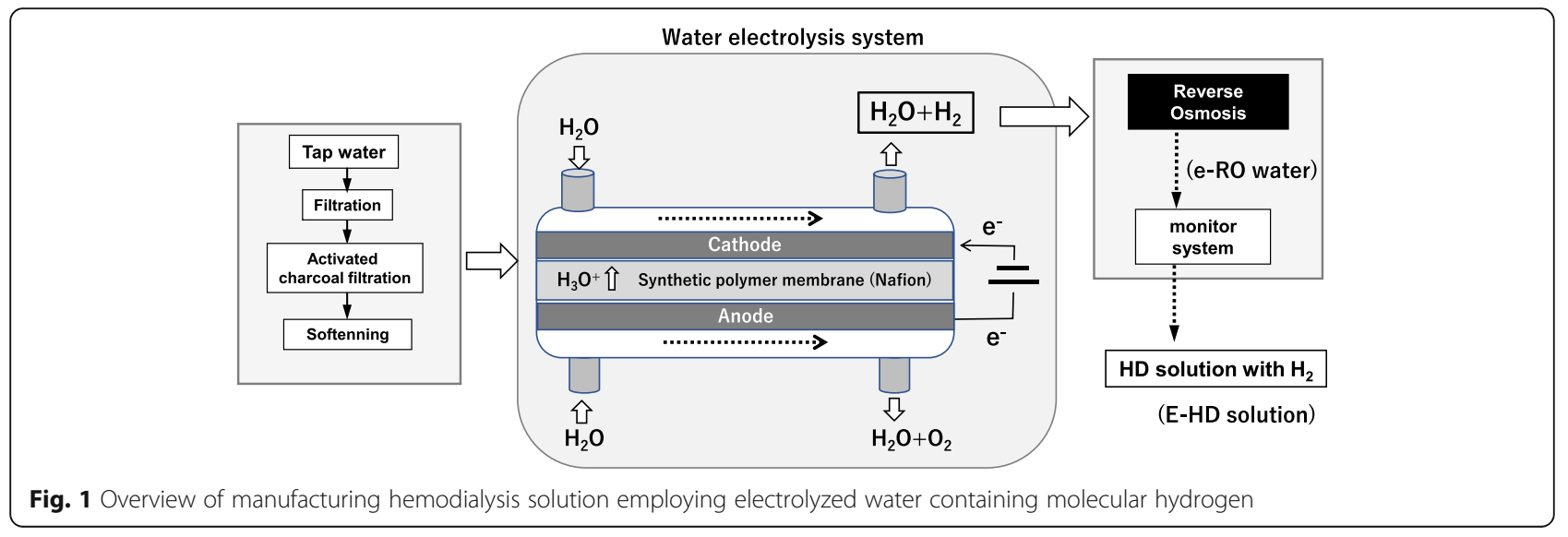


Table 2 Changes of redox parameters by hemodialysis and comparisons between CHD and EHD phases

\begin{tabular}{lllll}
\hline & & CHD phase & E-HD phase & $\boldsymbol{p}(\mathbf{C H D}$ vs. E-HD) \\
\hline MPO $(\mathrm{pg} / \mathrm{mL})$ & pre-HD & $427 \pm 197$ & $399 \pm 205$ & 0.413 \\
& post- & $656 \pm 289$ & $834 \pm 432$ & $<0.001$ \\
& $p$ (pre vs. post) & $<0.001$ & $<0.001$ & 0.065 \\
TRX $(\mathrm{ng} / \mathrm{mL})$ & pre-HD & $107.3 \pm 36.5$ & $114.0 \pm 44.2$ & $<0.001$ \\
& post- & $60.0 \pm 34.6$ & $76.2 \pm 29.9$ & $<0.001$ \\
MDA-a $(\mathrm{pmol} / \mathrm{mL})$ & pre vs. post) & $<0.001$ & $61.7 \pm 17.9$ & $<.030$ \\
& pre-HD & $59.0 \pm 21.4$ & $67.2 \pm 18.3$ & $<0.001$ \\
& post- & $72.9 \pm 21.2$ & 0.004 & \\
\hline
\end{tabular}

CHD Control HD; EHD HD employing electrolyzed water containing molecular hydrogen; MPO myeloperoxidase; RTX thioredoxin-1; MDA- $a$ malondialdehydeprotein adduct

were seen after the session of $\mathrm{HD}$, but the degrees of those changes differed significantly between CHD and E-HD phases. The increase in MDA-A was reduced, the decrease in TRX was reduced and the increase in MPO was accentuated in the E-HD phase as compared with the CHD phase.

Regarding the relationship between basal MPO level (pre-HD) and $\triangle \mathrm{MPO}$ (post-HD - pre-HD), no relationships were observed in the CHD phase, whereas a significant positive relationship was seen between the two parameters in the E-HD phase (Fig. 2).

In the CHD phase, significant positive correlations were identified between $\triangle \mathrm{MPO}$ and the percentage change in MDA-a between pre- and post-HD (\%MDAa) (Fig. $3 \mathrm{~A}$ ), and between $\triangle \mathrm{MPO}$ and the percentage change in TRX between pre- and post-HD (\%TRX) (Fig. $3 \mathrm{~B})$. In the E-HD phase, no correlation was found between $\triangle \mathrm{MPO}$ and \%MDA-a (Fig. 3C), while a significant positive correlation was observed between $\triangle \mathrm{MPO}$ and \%TRX (Fig. 3D).

In the CHD phase, NRS was $2.5 \pm 2.4$ on HD day. Patient reports on fatigue were as follows: 30 cases (47.6\%) in grade 1, $22(34.9 \%)$ in grade 2, $10(15.9 \%)$ in grade 3, and one (1.6\%) in grade 4 on HD days, and 39 cases
$(61.9 \%)$ in grade $1,22(34.9 \%)$ in grade $2,2(3.2 \%)$ in grade 3 , and none $(0.0 \%)$ in grade 4 on non-HD days.

Based on ROC curves, we determined the cut-off level for fatigue as NRS $\geq 3$ in the present study. According to basal levels in the CHD phase, 36 cases (57.1\%) were classified to the non-fatigue group and 27 cases $(42.9 \%)$ to the fatigue group. Clinical characteristics of the two groups are shown in Table 3. A faint but significant difference in serum albumin level was found between the two groups in the CHD phase (Table 3), and change in MDA-a due to HD was higher in the CHD phase (Table 4).

Logistic regression analysis was used to analyze associations between risk of fatigue (NRS $\geq 3$ ) and changes in redox parameters $(\triangle \mathrm{MPO}, \% \mathrm{TRX}, \% \mathrm{MDA}-\mathrm{a})$ for the CHD phase, after adjusting for serum albumin. Serum albumin and \%MDA-a were identified as independent factors for fatigue in the CHD phase (Table 5). While in E-HD phase, \%TRX was identified as independent factor for fatigue at 8 weeks (Table 6).

During the 8 weeks of observation as compared with basal NRS in the CHD phase, no changes were seen in the non-fatigue group, whereas significant decreases were observed at 2, 4, and 8 weeks, respectively (Fig. 4).
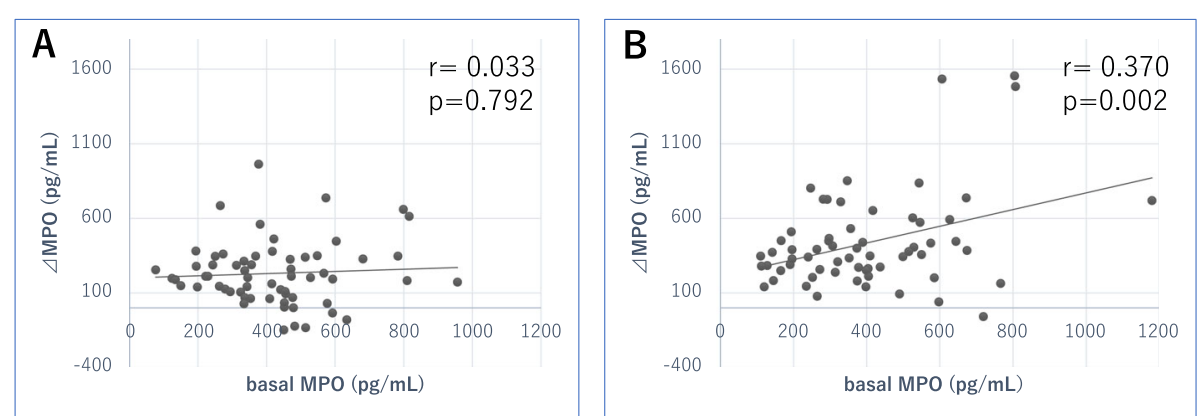

Fig. 2 Relationship between basal MPO levels (pre-HD) and $\triangle M P O$ by HD. A Control HD. B HD employing electrolyzed water (8th week). $\triangle M P O$ : post-HD level - pre-HD level. HD, hemodialysis; MPO, myeloperoxidase 
Table 3 Comparisons of patients basal background between the non-fatigue group and fatigue group

\begin{tabular}{llll}
\hline & Non-fatigue group (NRS $<3)$ & Fatigue group $(\mathbf{3}<=$ NRS) & $\boldsymbol{p}$ \\
\hline $\mathrm{N}$ & $36(57.1 \%)$ & $27(42.9 \%)$ & $73.1 \pm 9.3$ \\
Age (year old) & $71.7 \pm 9.4$ & $16(59.2 \%)$ & 0.581 \\
Male (\%) & $25(69.4 \%)$ & $10.0 \pm 10.0$ & 0.410 \\
HD vintage (years) & $9.5 \pm 7.0$ & $56.8 \pm 13.4$ & 0.843 \\
Dry weight (kg) & $60.6 \pm 14.9$ & $161 \pm 23 / 79 \pm 14$ & 0.313 \\
Pre-HD BP: SBP/DBP (mmHg) & $163 \pm 23 / 84 \pm 14$ & $152 \pm 28 / 76 \pm 12$ & $0.754 / 0.282$ \\
Post-HD BP: SBP/DBP (mmHg) & $146 \pm 21 / 83 \pm 14$ & $74 \pm 11$ & $0.347 / 0.876$ \\
Pre-HD HR (/min) & $74 \pm 9$ & $69 \pm 10$ & 0.809 \\
Post-HD HR (/min) & $74 \pm 13$ & $10(37.0 \%)$ & 0.313 \\
Presence of Diabetes mellitus & $17(47.2 \%)$ & $7(25.9 \%)$ & 0.430 \\
History CVD & $15(41.6 \%)$ & $3(11.1 \%)$ & 0.210 \\
Current cancer & $2(5.5 \%)$ & $9.7 \pm 1.7$ & 0.428 \\
Creatinine (mg/dL) & $10.3 \pm 2.3$ & $10.3 \pm 0.8$ & 0.261 \\
Hemoglobin (g/dL) & $10.6 \pm 1.2$ & $3.70 \pm 0.37$ & 0.299 \\
Albumin (g/dL) & $3.87 \pm 0.22$ & $0.53 \pm 0.82$ & 0.026 \\
CRP (mg/dL) & $0.37 \pm 0.52$ & 0.377 \\
\hline
\end{tabular}

HD hemodialysis; SBP systolic blood pressure; DBP diastolic blood pressure; HR heart rate; CVD cardiovascular disease; CRP C-reactive protein
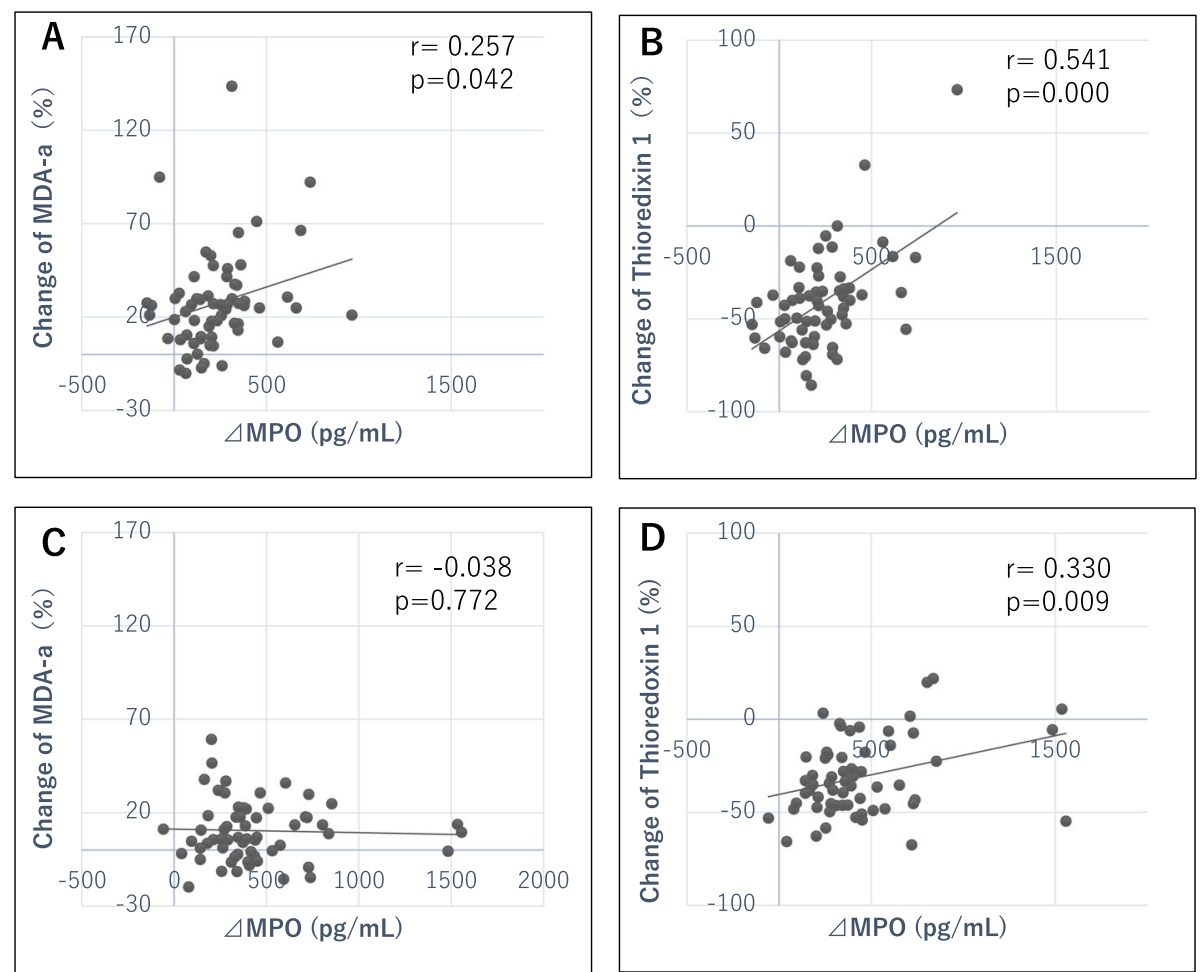

Fig. 3 Relationship between $\triangle M P O$ by HD and percentage changes in redox molecules (\%) by HD. A, B Control HD (CHD phase). C, D E-HD (EHD phase). Percentage change in molecule (\%): (post-HD level - pre-HD level)/pre-HD level $\times 100 . \triangle M P O:$ post-HD level - pre-HD level. HD, hemodialysis; MPO, myeloperoxidase; MDA-a, malondialdehyde-protein adduct 
Table 4 Comparisons of change of redox parameters by hemodyalysis between CHD and E-HD phases, and between the nonfatigue group and fatigue group

\begin{tabular}{lllll}
\hline & All & Non-fatigue group & Fatigue group & \multicolumn{1}{c}{} \\
& $(\boldsymbol{n}=\mathbf{6 3})$ & $(\boldsymbol{n}=\mathbf{3 6})$ & $\boldsymbol{( n = 2 7 )}$ & Non-Fatigue vs. Fatigue \\
\hline $\mathrm{CHD}$ & $+233.1 \pm 206.2$ & $+245.0 \pm 196.5$ & $+216.1 \pm 230.2$ & 0.602 \\
$\mathrm{EHD}$ & $+434.2 \pm 312.4$ & $+480.1 \pm 330.1$ & 0.206 \\
$p$ & $<0.001$ & $<0.001$ & 0.002 & \\
$\mathrm{CHD}$ & $-41.7 \pm 25.3$ & $-37.3 \pm 29.5$ & $-43.5 \pm 19.1$ & 0.626 \\
$\mathrm{EHD}$ & $-31.1 \pm 20.3$ & $-29.5 \pm 22.4$ & $-33.1 \pm 17.4$ & 0.509 \\
$p$ & 0.004 & 0.058 & 0.017 & \\
$\mathrm{CHD}$ & $+27.4 \pm 26.3$ & $+22.1 \pm 16.6$ & $+34.5 \pm 34.5$ & 0.063 \\
$\mathrm{EHD}$ & $+10.2 \pm 15.8$ & $+8.4 \pm 15.7$ & $+12.7 \pm 16.0$ & 0.297 \\
$p$ & $<0.001$ & $<0.001$ & 0.001 & \\
\hline
\end{tabular}

In the E-HD phase, both groups showed similar changes in redox parameters (Table 4).

\section{Discussion}

The present study aimed to examine the primary mechanisms underlying the anti-oxidative actions of E-HD, and their possible effects in ameliorating HD-related fatigue. The study used a single-arm, prospective, observational design, and the main findings are summarized as follows. In the CHD phase, increases in serum MPO accompanied increases in oxidative product (MDA-a) and anti-oxidant molecules (TRX), whereas in the E-HD phase, increases in MPO were accentuated during HD, but changes in MDA-a and TRX were both ameliorated as compared with the CHD phase. During the 8 weeks of observation, patients with HD-related fatigue displayed significant decreases in fatigue score.

Enhanced oxidative stress in HD patients could lead to the development of micro-inflammation, which would potentially play a role in cardio-cerebrovascular events and dialysis-related complications [11]. Among factors exacerbating oxidative stress, bio-incompatibility of the HD system could be a substantial contributor. With the current E-HD system, greater increases in MPO during $\mathrm{HD}$ were noted. MPO is involved in the generation of ROS in the presence of $\mathrm{H}_{2} \mathrm{O}_{2}[10,13]$, and thus may be associated with exaggerated oxidative stress when viewed in isolation.

The significant positive correlation between $\triangle \mathrm{MPO}$ and increased oxidative products in the CHD phase may well support this notion. Interestingly, concentrations of the anti-oxidant molecule TRX (molecular weight, $12 \mathrm{kDa}$ ) decreased significantly with $\mathrm{HD}$, probably due to partial loss during the HD process, while a positive correlation between $\triangle \mathrm{MPO}$ and \%TRX was apparent in the CHD phase. This suggests a biological response counter-regulating the enhanced creation of reactive oxygen species (ROS). We speculate that increased levels of MPO would induce oxidative processes due to activation of nuclear factor- $\mathrm{kB}$ (NFKB), but oxidative stress would simultaneously activate the Nrf2 (nuclear factor erythroid 2 (NF-E2)-related factor 2)-Keap1 (Kelch-like ECH-associated protein 1) signaling to generate anti-oxidants [29]. The exact profiles of Nrf2 and NFkB activation during HD need to be clarified.

Unexpectedly, MPO levels during HD were even more elevated in E-HD than in CHD. Several mechanisms could be involved with MPO release during HD, such as the physiological responses of neutrophils with decreased ionic $\mathrm{Ca}$ in the cell [30], removal of degranulation suppressors, angiogenin from blood during HD [31], and non-physiological neutrophil bursts, such as with mechanical damage from HD [8]. We have previously reported that the respiratory burst function of neutrophils is much better preserved in electrolyzed water-treated human neutrophils as compared with controls [23]. Taking these observations together, enhanced MPO release may reflect better neutrophil viability with the use of electrolyzed water-based dialysis solution.

Table 5 Multiple logistic regression analysis for the presence of fatigue at the CHD-phase

\begin{tabular}{llll}
\hline Variable & Odd ratio & $\mathbf{9 5 \% C l}$ & $\boldsymbol{p}$ value \\
\hline$\triangle \mathrm{MPO}$ & 0.998 & $(0.995-1.002)$ & 0.296 \\
$\triangle \mathrm{TRX} \%$ & 1.001 & $(0.974-1.029)$ & 0.998 \\
$\triangle \mathrm{MDA}-\mathrm{a} \%$ & 1.028 & $(1.001-1.056)$ & 0.038 \\
Albumin & 0.075 & $(0.008-0.695)$ & 0.023 \\
\hline
\end{tabular}


Table 6 Multiple logistic regression analysis for the presence of fatigue at the EHD-phase

\begin{tabular}{llll}
\hline Variable & Odd ratio & 95\%Cl & $p$ value \\
\hline$\triangle \mathrm{MPO}$ & 0.998 & $(0.995-1.001)$ & 0.140 \\
$\triangle \mathrm{TRX} \%$ & 1.036 & $(1.003-1.069)$ & 0.030 \\
$\triangle \mathrm{MDA}-\mathrm{a} \%$ & 0.991 & $(0.956-1.027)$ & 0.613 \\
Albumin & 0.545 & $(0.094-3.151)$ & 0.498 \\
\hline
\end{tabular}

Regarding the changes of redox parameters during $\mathrm{HD}$, there were significant increases in MPO and MDA-a, and decreases in TRX in CHD and E-HD phases. Interestingly, increases of MDA-a in the EHD were significantly lower than those of CHD, while decreases in TRX in E-HD were significantly higher than those of CHD (Table 2), although increases of MPO by HD, which is supposed to be a surrogate marker for oxidative stress, were significantly higher in E-HD than those in CHD. Upon those changes of MDA-a and TRX, it seems that E-HD may directly or indirectly suppress ROS and activate or preserve antioxidant system during HD. Nevertheless, as compared with those of the CHD phase, the marginal but significant increases of MDA-a in the pre-HD levels at the E-HD phase (8th week), may indicate the



exaggerated oxidative stress by E-HD, whereas the higher TRX of the pre-HD levels at the E-HD phase may indicate the potentiation of anti-oxidative system by E-HD (Table 2). At present, we cannot explain the mechanism of this intriguing finding clearly. Instead, our working hypothesis over the post-HD fatigue and the therapeutic contribution by E-HD are shown in Fig. 5.

The present study assessed the extent of fatigue using patient reports. We defined "clinically relevant fatigue" as subjective symptoms reflecting reduced activities of daily living. Based on data from the CHD phase, 27 of the 63 patients $(42.9 \%)$ were classified to the fatigue group. Lower serum albumin and higher MAD-a elevation during HD were identified as risk factors for the presence of fatigue in the CHD phase. During the 8-week observation, NRS scores in the fatigue group were significantly improved from 2 weeks after the introduction of E-HD, whereas no clinically significant changes were observed in the non-fatigue group. Regarding redox profiles between fatigue and non-fatigue groups, no differences were observed between groups in the E-HD phase. These results may indicate a pathological role of enhanced oxidative stress in the development of fatigue after HD, and EHD may be of particular benefit in ameliorating subjective symptoms of fatigue among patients affected in this manner.

Several limitations to the present study need to be considered when interpreting the results. First, we did not correct for measured test molecules, and levels in the post-HD period may thus have been overestimated. Post-HD levels have been speculated to be nearly $10 \%$ lower if the fluid were not removed. Nevertheless, the significant differences between the CHD and E-HD phases at the respective time-points appear likely to be robust. Second, we only measured redox parameters at two time-points, pre- and post$\mathrm{HD}$, and the temporal course of changes after HD were unclear. Although MPO levels post-HD were significantly higher in the E-HD phase than in the CHD phase, this result does not confirm a larger AUC of MPO during the periods after HD. This crucial issue regarding peak MPO levels and the AUC after HD needs to be clarified. Third, the present single-arm observational study was unable to answer 


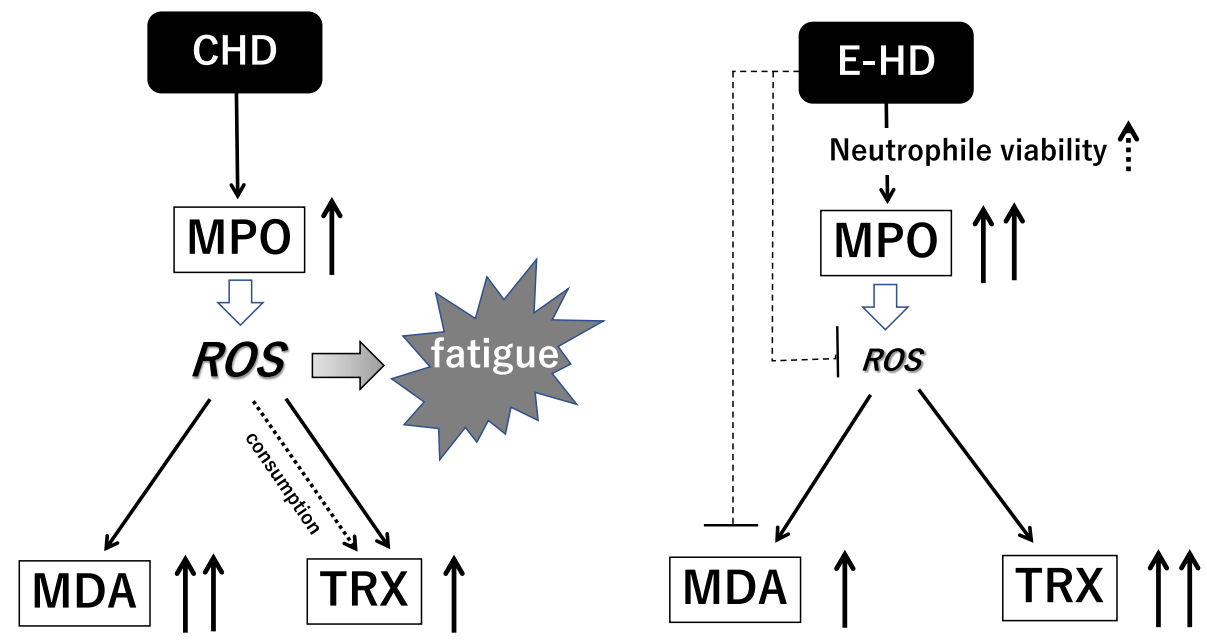

Fig. 5 Working hypothesis. Our hypothesis is that not MPO but ROS may play a primary role for the development of HD-related fatigue. ROS itself cannot be measured at routine examinations, thus, the change of MDA-a level is employed as a surrogate marker for the intensity of ROS in the clinical setting. The result that $\triangle \mathrm{MDA}$-a was identified as an independent risk factor for the presence of fatigue in CHD (Table 5), may well support this notion. In this regard, increases of MPO by E-HD does not necessarily reflect the enhanced oxidative stress and may be the result of improved neutrophil viability. The exact mechanisms leading to suppression of oxidative stress and enhancement of anti-oxidation have remained unclear. However, we suppose the following possibilities. Regarding the mechanisms underlying decreased MDA-a, $\mathrm{H}_{2}$ in the dialysis solution may directly quench radical oxygen species, thereby decreasing adduct production, or E-HD may increase the viability of cells that scavenge the MDA-a, thereby shortening the disappearance time. Regarding the increase in thioredoxin, decreased consumption/production of thioredoxin due to less oxidative stress, or increased viability of the cells producing thioredoxin, may be involved with the phenomenon. C-HD, standard hemodialysis; E-HD, hemodialysis employing electrolyzed water; MPO, myeloperoxidase; ROS, reactive oxygen species; MDA-a, malondialdehyde-protein adduct; TRX, thioredoxin-1

whether E-HD can suppress subjective fatigue, or whether the oxidative stress from HD plays a role in the development of HD-related clinical fatigue. In the future, randomized control studies are needed to clarify these issues. Last, evaluation on fatigue used a subjective, patient-reported method, and thus lacks consistency. The development of novel parameters based on objective systems is expected.

\section{Conclusion}

E-HD appears to ameliorate oxidative stress and increase anti-oxidant systems during HD, suggesting improved bio-compatibility of the HD system. E-HD could benefit patients with HD-related fatigue, but the mechanisms in relation to oxidative stress remain to be elucidated.

\section{Abbreviations}

HD: Hemodialysis; MPO: Myeloperoxidase; E-HD: Hemodialysis employing electrolyzed water containing molecular hydrogen $(\mathrm{H} 2)$; TRX: Thioredoxin 1; MDA-a: Malondialdehyde-protein adduct; NSR: Numerical rating scale; NFkB: Nuclear factor-kB; Nrf2: Nuclear factor erythroid 2 (NF-E2)-related factor 2; Keap1: Kelch-like ECH-associated protein 1; ROS: Reactive oxygen species

\section{Acknowledgements}

The authors would like to thank the staff of Yokodai central clinic and Ms Takahashi (secretary of Tohoku University Hospital, Research Division) for their help in collecting data and samples of patients.

\section{Declarations}

\section{Authors' contributions}

$\mathrm{HS}, \mathrm{NK}, \mathrm{KS}$, and MN designed the study and analyzed the data. MN and KS drafted the manuscript. HS collected and entered data. TI, YK, NK, and MY contributed to the data acquisition and interpretation. All authors read and approved the final manuscript.

Funding

No funding was received.

\section{Availability of data and materials}

The datasets used and/or analyzed in the current study are available from the corresponding author on reasonable request.

\section{Ethics approval and consent to participate}

The study protocol was fully approved by the ethics committee of Kanagawa Medical Association Ethics Review Committee (October 10, 2019), and performed in accordance with the Declaration of Helsinki. Informed consent was obtained from all individual participants.

Consent for publication

Not applicable.

\section{Competing interests}

The authors declared no competing interests.

\section{Author details}

${ }^{1}$ Houshinkai Yokodai Central Clinic, Yokohama, Japan. ${ }^{2}$ Department of Nephrology and Hypertension, Saiseikai Yokohama-shi Nanbu Hospital, Yokohama, Japan. ${ }^{3}$ Houshinkai Toshin Clinic, Yokohama, Japan. ${ }^{4}$ Department of Nephrology, Yokohama City Minato Red Cross Hospital, Yokohama, Japan. ${ }^{5}$ Kidney Center, St Luke's International Hospital, 9-1 Akashicho, Chuo-ku, 
Tokyo 1048560, Japan. ${ }^{6}$ Research Division of Dialysis Chronic Kidney Disease, Tohoku University Hospital, Sendai, Japan.

\section{Received: 17 March 2021 Accepted: 3 June 2021}

Published online: 25 June 2021

\section{References}

1. Hörl WH. Hemodialysis membranes: interleukins, biocompatibility, and middle molecules. J Am Soc Nephrol. 2002;13(Suppl 1):S62-71. https://doi. org/10.1681/ASN.V13suppl_1s62.

2. Hakim RM. Clinical implications of biocompatibility in blood purifcation membranes. Nephrol Dial Transplant. 2000;15(Suppl 2):16-20. https://doi. org/10.1093/ndt/15.suppl_1.16.

3. Bommer J, Jaber BL. Ultrapure dialysate: facts and myths. Semin Dial. 2006; 19(2):115-9. https://doi.org/10.1111/j.1525-139X.2006.00136.x.

4. Bouman CS, van Olden RW, Stoutenbeek CP. Cytokine fltration and adsorption during pre- and postdilution hemofltration in four diferent membranes. Blood Purif. 1998;16(5):261-8. https://doi.org/10.1159/ 000014343 .

5. Andreoli MCC, Dalboni MA, Watanabe R, Manfredi SR, Canziani MEF, Kallás $E G$, et al. Impact of dialyzer membrane on apoptosis and function of polymorphonuclear cells and cytokine synthesis by peripheral blood mononuclear cells in hemodialysis patients. Artif Organs. 2007;31(12):88792. https://doi.org/10.1111/j.1525-1594.2007.00485.x.

6. Wu CC, Liao TN, Lu KC, Chen JS, Chu P, Lin SH, et al. Apoptotic markers on lymphocytes and monocytes are unchanged during single hemodialysis sessions using either regenerated cellulose or polysulfone membranes. Clin Nephrol. 2005;64(3):198-204. https://doi.org/10.5414/CNP64198.

7. Nakayama M, Tani Y, Zhu WJ, Watanabe K, Yokoyama K, Fukagawa M, et al. Oral ferric citrate hydrate associated with less oxidative stress than intravenous saccharated ferric oxide. Kidney Int Rep. 2018;3(2):364-73. https://doi.org/10.1016/.ekir.2017.10.016.

8. Fukushi T, Yamamoto T, Yoshida M, Fujikura E, Miyazaki M, Nakayama M. Enhanced neutrophil apoptosis accompanying myeloperoxidase release during hemodialysis. Sci Rep. 2020;10(1):21747. https://doi.org/10.1038/s41 598-020-78742-z.

9. Honda H, Ueda M, Kojima S, Mashiba S, Hirai Y, Hosaka N, et al. Assessment of myeloperoxidase and oxidative alpha1-antitrypsin in patients on hemodialysis. Clin J Am Soc Nephrol. 2009;4(1):142-51. https://doi.org/1 0.2215/CJN.02280508.

10. Wu CC, Chen JS, Wu WM, Liao TN, Chu P, Lin SH, et al. Myeloperoxidase serves as a marker of oxidative stress during single haemodialysis session using two different biocompatible dialysis membranes. Nephrol Dial Transplant. 2005;20(6):1134-9. https://doi.org/10.1093/ndt/gfh764.

11. Himmelfarb J, Stenvinkel P, Ikizler TA, Hakim RM. The elephant in uremia: oxidant stress as a unifying concept of cardiovascular disease in uremia. Kidney Int. 2002;62(5):1524-38. https://doi.org/10.1046/j.1523-1 755.2002.00600.x.

12. Kalantar-Zadeh K, Brennan ML, Hazen SL. Serum myeloperoxidase and mortality in maintenance hemodialysis patients. Am J Kidney Dis. 2006;48(1): 59-68. https://doi.org/10.1053/j.ajkd.2006.03.047.

13. Borawski J. Myeloperoxidase as a marker of hemodialysis biocompatibility and oxidative stress: the underestimated modifying effects of heparin. Am J Kidney Dis. 2006;47(1):37-41. https://doi.org/10.1053/j.ajkd.2005.10.001.

14. Jhamb M, Weisbord SD, Steel JL, Unruh M. Fatigue in patients receiving maintenance dialysis: a review of definitions, measures, and contributing factors. Am J Kidney Dis. 2008;52(2):353-65. https://doi.org/10.1053/j.ajkd.2 008.05.005.

15. Ju A, Unruh ML, Davison SN, Dapueto J, Dew MA, Fluck R, et al. PatientReported Outcome Measures for Fatigue in Patients on Hemodialysis: A Systematic Review. Am J Kidney Dis. 2018;71(3):327-43. https://doi.org/10.1 053/.j.jkd.2017.08.019.

16. Jhamb M, Argyropoulos C, Steel JL, Plantinga L, Wu AW, Fink NE, et al. Correlates and outcomes of fatigue among incident dialysis patients. Clin J Am Soc Nephrol. 2009:4(11):1779-86. https://doi.org/10.2215/CJN.00190109.

17. Jhamb M, Pike F, Ramer S, Argyropoulos C, Steel J, Dew MA, et al. Impact of fatigue on outcomes in the hemodialysis (HEMO) study. Am J Nephrol. 2011;33(6):515-23. https://doi.org/10.1159/000328004.

18. Bossola M, Di Stasio E, Antocicco M, Panico L, Pepe G, Tazza L. Fatigue Is Associated with Increased Risk of Mortality in Patients on
Chronic Hemodialysis. Nephron. 2015;130(2):113-8. https://doi.org/1 $0.1159 / 000430827$

19. Koyama H, Fukuda S, Shoji T, Inaba M, Tsujimoto Y, Tabata T, et al. Fatigue is a predictor for cardiovascular outcomes in patients undergoing hemodialysis. Clin J Am Soc Nephrol. 2010;5(4):659-66. https://doi.org/1 0.2215/CJN.08151109.

20. Davenport A, Guirguis A, Almond M, Day C, Chilcot J, Maria Da Silva Gane M.D.S, et al. Postdialysis recovery time is extended in patients with greater self-reported depression screening questionnaire scores. Hemodial Int 2018; 22(3):369-376, DOl: https://doi.org/10.1111/hdi.12642.

21. Fujii H, Koyama H, Fukuda S, Tokai H, Tajima S, Koizumi J, et al. Autonomic function is associated with health-related quality of life in patients with end-stage renal disease: a case-control study. J Ren Nutr. 2013;23(5):340-7. https://doi.org/10.1053/j.jr. 2012.12.008.

22. Alvarez L, Brown D, Hu D, Chertow GM, Vassalotti JA, Prichard S, et al. Intradialytic Symptoms and Recovery Time in Patients on Thrice-Weekly InCenter Hemodialysis: A Cross-sectional Online Survey. Kidney Med. 2019; 2(2):125-30. https://doi.org/10.1016/j.xkme.2019.10.010.

23. Nakayama M, Kabayama S, Terawaki H, Nakayama K, Kato K, Sato T, et al. Less-oxidative hemodialysis solution rendered by cathode-side application of electrolyzed water. Hemodial Int. 2007;11(3):322-7. https://doi.org/1 0.1111/j.1542-4758.2007.00187.x.

24. Nakayama M, Kabayama S, Nakano H, Zhu WJ, Terawaki H, Nakayama K, et al. Biological effects of electrolyzed water in hemodialysis. Nephron Clin Pract. 2009;112(1):c9-15. https://doi.org/10.1159/000210569.

25. Terawaki H, Zhu WJ, Matsuyama Y, Terada T, Takahashi Y, Sakurai K, et al. Effect of a hydrogen ( $\mathrm{H} 2)$-enriched solution on the albumin redox of hemodialysis patients. Hemodial Int. 2014;18(2):459-66. https://doi.org/1 $0.1111 /$ hdi.12112.

26. Nakayama M, Nakano H, Hamada H, Itami N, Nakazawa R, Ito S. A novel bioactive haemodialysis system using dissolved dihydrogen $(\mathrm{H} 2)$ produced by water electrolysis: a clinical trial. Nephrol Dial Transplant. 2010;25(9): 3026-33. https://doi.org/10.1093/ndt/gfq196.

27. Namayama M, Itami N, Suzuki H, Hamada H, Yamamoto R, Tsunoda K, et al. Novel haemodialysis (HD) treatment employing molecular hydrogen $(\mathrm{H} 2)$ enriched dialysis solution improves prognosis of chronic dialysis patients: a prospective observational study. Sci Rep. 2018;8(1):254. https://doi.org/10.1 038/s41598-017-18537-X.

28. Nakayama M, Itami N, Suzuki H, Hamada H, Osaka N, Yamamoto R, et al. Possible clinical effects of molecular hydrogen $(\mathrm{H} 2)$ delivery during hemodialysis in chronic dialysis patients: Interim analysis in a 12 month observation. PLoS One. 2017;12(9):e0184535. https://doi.org/10.1371/journal. pone. 0184535 .

29. Itoh K, Mimura J, Yamamoto M. Discovery of the negative regulator of $\mathrm{Nrf2}$, Keap1: a historical overview. Antioxid Redox Signal. 2010;13(11):1665-78. https://doi.org/10.1089/ars.2010.3222.

30. Bos JC, Grooteman MP, van Houte AJ, Schoorl M, van Limbeek J, Nubé MJ. Low polymorphonuclear cell degranulation during citrate anticoagulation: a comparison between citrate and heparin dialysis. Nephrol Dial Transplant. 1997;12(7):1387-93. https://doi.org/10.1093/ndt/12.7.1387.

31. Schmaldienst S, Oberpichler A, Tschesche H, Hörl WH. Angiogenin: a novel inhibitor of neutrophil lactoferrin release during extracorporeal circulation. Kidney Blood Press Res. 2003;26(2):107-12. https://doi.org/10.1159/ 000070992.

\section{Publisher's Note}

Springer Nature remains neutral with regard to jurisdictional claims in published maps and institutional affiliations. 